\title{
Locating a Circular Biochemical Source: Modeling and Control
}

\author{
Panos Tzanos and Miloš Žefran
}

\begin{abstract}
This paper applies the modified Fisher Information Matrix (FIM) motion algorithm previously proposed by the authors to the task of locating a circular biochemical source. We develop the diffusion model for a circular source and perform control theoretic analysis of the resulting FIM motion algorithm. While in our previous work we established that the source location is an equilibrium point of the system, in the present paper we show that due to the consistency of the maximum likelihood (ML) estimator, the equilibrium point is unique. Simulations are presented that compare our motion algorithm to conventional concentration gradient motion algorithms. The simulations confirm that by using our motion algorithm, the circular biochemical source is located with a high degree of accuracy.
\end{abstract}

\section{INTRODUCTION}

Biochemical source localization is an important task for various fields such as public safety, environmental monitoring, and the military. Systems using sensors that can detect low concentration vapors have found use in applications such as landmine detection and localization [1], and the monitoring of the ocean's dynamic physical characteristics and chemical distributions [2]. These sensors also have the potential to be used in other applications such as detection of biochemical weapons and drugs, sensing leakage of hazardous chemicals, pollution sensing and environmental studies [3], [4].

It is envisioned that in the near future many of the previously mentioned sensing tasks will be performed by using groups of robots working together through ad-hoc communication networks due to technological advances in networking and the miniaturization of electromechanical systems [5]. However, the coordination algorithms for such mobile sensing networks are difficult to develop since they must conform to the spatially-distributed nature and limited communication capabilities of the network. A different approach to network communication known as distributed or localized networking takes advantage of the physically distributed nature of the sensors by designing coordination algorithms that allow sensor nodes to only communicate with other nodes within some neighborhood. These networks have the advantage that the communication overhead scales well with increases in network size, and they are robust to failure due to the lack of a central data processing node [6].

Previous work on the problem of tracing an odor plume to its source has been done by [7]-[10]. Many of the early

This research was supported in part by NSF grants IIS-0093581, CCR0330342, and CMS-0600658.

P. Tzanos and M. Žefran are with the Department of Electrical and Computer Engineering, University of Illinois at Chicago, Chicago, IL 60607, USA. Email: \{ptzanol,mzefran $\} @ u i c . e d u$. motion algorithms were based on the observations of the motions of animals following a scent [11]-[13]. One of the approaches that came out of this work was the use of the concentration gradient to find the source [14]. In [15], an alternative approach was proposed, where the robot moves so that in each step the information that its sensors provide is maximized. The algorithm outperforms concentration gradient based schemes, but it requires more sophisticated modeling and estimation.

Using this information based approach, [15] describes how a single vehicle equipped with vapor sensors can estimate the location of a source emitting vapor at a constant rate in an infinite volume. Estimation is performed using maximum likelihood (ML) estimator, and the vehicle is controlled by moving the vehicle in the opposite direction of the gradient of the Cramér-Rao bound (CRB) on the location error. Since ML estimates are asymptotically efficient (their variance approaches the CRB as the data length goes to infinity), it is expected that the actual accuracy of the estimation will be close to the CRB. Hence, by minimizing the CRB the actual estimation error is expected to be minimized. The use of the CRB or Fisher information (the inverse of the CRB) as an optimality criterion for other control problems has been explored in [16] and [17]. Other authors have used Kalman filter estimation [18], or Levenberg-Marquardt optimization [19] for estimating unknown parameters. However, with such methods, there is no guarantee of achieving the lowest possible estimator variance.

The diffusion of a chemical in a medium is well understood mathematically [20], [21]. Previous authors working on this subject [4], [15], [22] have assumed the biochemical source to be a point source. While such an assumption simplifies the mathematical modeling of the chemical concentration considerably, assuming the chemical source to have zero volume is unrealistic, and at the same time creates difficulties in stability analysis due to the singularity in the concentration function at the source location. In [23] the authors attempt to model the diffusion from a circular source through a function that resembles in shape the theoretical concentration function from such a source. They go on to develop another information based motion algorithm named the modified Fisher Information Matrix (FIM) motion algorithm and show that for any concentration function with a maximum at the source location, the motion algorithm has an equilibrium point at the source location. In this paper we develop the concentration model for a circular source through the theory of molecular diffusion. This will make our problem more challenging numerically, but on the other hand the model is more realistic while all the stability results of [23] still apply. 
Furthermore, we use the ML estimator's property of consistency (the ML estimate approaches the true value as the data length approaches infinity), to argue that the only equilibrium point of the system is the center of the circular source. It is shown through simulations that with the use of the modified FIM motion algorithm the vapor source is located with a high degree of accuracy and outperforms conventional gradient algorithm techniques.

\section{Mathematical Modeling}

In this section we describe the physical and measurement models for the case of a circular source in an infinite volume emitting vapor at a constant rate.

\section{A. Physical Model}

Let $c(\vec{r}, t)$ be the concentration of a diffusing substance at a point $\vec{r}=(x, y, z)$ at time $t$. Assuming that the diffusivity $\kappa$ of the medium is space-invariant, the evolution of $c(\vec{r}, t)$ is described by the classic diffusion equation for a source free volume

$$
\frac{\partial c}{\partial t}=\kappa \nabla^{2} c
$$

For a point source at $\vec{r}_{\circ}=\left(x_{\circ}, y_{\circ}, z_{\circ}\right)$ in an infinite medium, releasing a diffusing substance at a constant rate of $\mu$, starting at time $t_{\circ}$, the solution to (1) is given by [20]

$$
c(\vec{r}, t)=\frac{\mu}{4 \pi \kappa\left|\vec{r}-\vec{r}_{\circ}\right|} \operatorname{erfc}\left(\frac{\left|\vec{r}-\vec{r}_{\circ}\right|}{2 \sqrt{\kappa\left(t-t_{\circ}\right)}}\right)
$$

where $\operatorname{erfc}(x)=(2 / \sqrt{\pi}) \int_{x}^{\infty} e^{-y^{2}} d y$ is the complementary error function. To find the solution for a circular source, we must integrate (2) over the area of the source. Let $\vec{r}^{\prime}=$ $\left(x^{\prime}, y^{\prime}\right)$ be a point on the source. We will assume throughout this paper that the source is located on the ground $(z=$ 0 ), and likewise all points of observation. Then the solution to (1) for a circular source can be written as

$c(\vec{r}, t)=\int_{0}^{2 \pi} \int_{0}^{a} \frac{\mu}{4 \pi \kappa\left|\vec{r}-\vec{\rho}_{\circ}\right|} \operatorname{erfc}\left(\frac{\left|\vec{r}-\vec{\rho}_{\circ}\right|}{2 \sqrt{\kappa\left(t-t_{\circ}\right)}}\right) \rho d \rho d \phi$

where $\vec{\rho}_{\circ}=\left(x_{\circ}+\rho \cos (\phi), y_{\circ}+\rho \sin (\phi)\right)$ and $a$ is the radius of the source. Unlike the solution for the point source, the concentration function in (3) does not have a singularity at $\vec{r}=\vec{r}_{\mathrm{o}}$, as illustrated in Figure 1. This is important, because otherwise the stability analysis at the center of the source would not be possible.

\section{B. Measurement Model}

Although chemical sensors are designed to be highly sensitive to the substance of interest, and as insensitive as possible to undesired substances, no sensor can be completely insensitive to foreign materials [15]. It is therefore reasonable to model the response of the sensor located at the point $\vec{r}$ by

$$
y(\vec{r}, t)=c(\vec{r}, t)+b(\vec{r}, t)+e(\vec{r}, t)
$$

where $c(\vec{r}, t)$ is the concentration of the substance of interest given by $(3), b(\vec{r}, t)$ is the bias or "clutter" term, representing

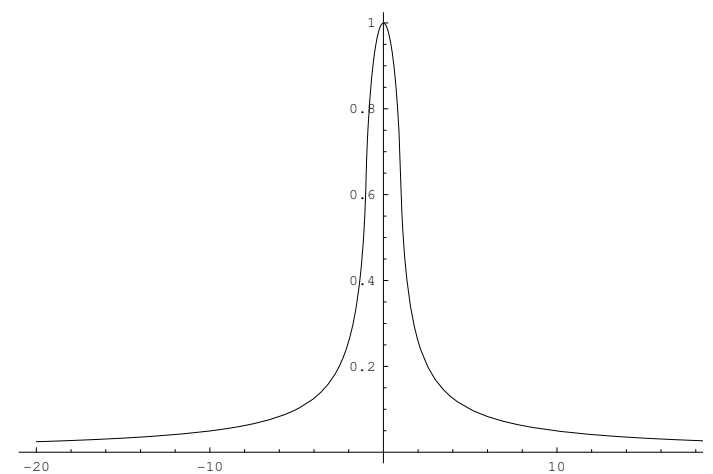

Fig. 1. A realization of the one dimensional cross-section of the concentration function given in Eq. (3)

the sensor's response to foreign substances, and $e(\vec{r}, t)$ is the sensor's noise. The source location $\vec{r}_{\circ}$, its intensity $\mu$, the diffusivity $\kappa$, and the release time $t_{\circ}$ are all unknown parameters. The bias term is assumed to be unknown, time invariant, and uniformly distributed in space. This means the substances interfering with the desired measurement are modeled as being in a steady state and the result of many sources, so they have reached both temporal and spatial equilibrium. The noise is modeled as Gaussian with zero mean and unknown variance $\sigma_{e}^{2}$, uncorrelated in time, and independent from sensor to sensor. The assumption of Gaussian noise is not quite physical since $y(\vec{r}(t), t)$ must be a non-negative quantity. However for $\sigma_{e}$ much smaller than $c(\vec{r}(t), t)+b(\vec{r}(t), t)$, the Gaussian model is a reasonable approximation.

\section{PARAMETER Estimation}

Here we review the maximum likelihood approach to estimating the unknown parameters $\Psi=\left[\vec{r}_{\circ}, \kappa, t_{\circ}, \mu, b, \sigma_{e}^{2}\right]^{T}$ and the Cramér-Rao lower bound. This is presented in detail in [4].

Let the available measurements be $\left\{y\left(\vec{r}\left(t_{k}\right), t_{k}\right), 1 \leq k \leq\right.$ $p\}$, where $p \geq 2$, and partition the parameter vector as $\Psi=$ $\left[\theta^{T}, \mathbf{x}^{T}, \sigma_{e}^{2}\right]^{T}$, where $\theta=\left[\vec{r}_{\circ}, \kappa, t_{\circ}\right]^{T}$ and $\mathbf{x}=[\mu, b]^{T}$. With this notation the measurements can be transformed into the following vector form

$$
\mathbf{y}=A(\theta) \mathbf{x}+\mathbf{e}
$$

where $\mathbf{y}$ and $\mathbf{e}$ are $p$-dimensional vectors whose $k$-th components are $y\left(\vec{r}\left(t_{k}\right), t_{k}\right)$ and $e\left(\vec{r}\left(t_{k}\right), t_{k}\right)$ respectively, and $A(\theta)$ is a $p \times 2$ matrix whose $k$-th row is given by

$$
\left[\int_{0}^{2 \pi} \int_{0}^{a} \frac{\rho}{4 \pi \kappa\left|\vec{r}\left(t_{k}\right)-\vec{\rho}_{\circ}\right|} \operatorname{erfc}\left(\frac{\left|\vec{r}\left(t_{k}\right)-\vec{\rho}_{\circ}\right|}{2 \sqrt{\kappa\left(t_{k}-t_{\circ}\right)}}\right) \rho d \rho d \phi, 1\right] \text {. }
$$

The maximum likelihood estimates $\hat{\theta}, \hat{\mathbf{x}}$, and $\hat{\sigma}_{e}^{2}$ were computed in [4] and are

$$
\begin{aligned}
& \hat{\theta}=\arg \max _{\theta}\left\{\mathbf{y}^{T} P_{A}(\theta) \mathbf{y}\right\} \\
& \hat{\mathbf{x}}=\left[A^{T}(\hat{\theta}) A(\hat{\theta})\right]^{-1} A^{T}(\hat{\theta}) \mathbf{y} \\
& \hat{\sigma}_{e}^{2}=(p)^{-1} \mathbf{y}^{T} P_{A}^{\perp}(\hat{\theta}) \mathbf{y}
\end{aligned}
$$


where $P_{A}(\theta)=A(\theta)\left[A^{T}(\theta) A(\theta)\right]^{-1} A^{T}(\theta)$ is the projection matrix onto the column space of $A(\theta)$ and $P_{A}^{\perp}(\theta)=I-$ $P_{A}(\theta)$ is the complementary projection matrix. From Eq. (6) it is seen that the ML estimate $\hat{\theta}$ is the $\theta$ that maximizes the projection of the column space of $A(\theta)$ onto the data vector $\mathbf{y}$ and vice versa.

By writing $A(\theta)$ in terms of its two columns as $[\mathbf{a}(\theta), \mathbf{u}]$ where $\mathbf{u}$ is a vector of 1's, the CRB's for unbiased estimates of the parameters are

$$
\begin{aligned}
& \operatorname{CRB}(\theta)=\frac{\sigma_{e}^{2}}{\mu^{2}}\left\{D^{T}(\theta) P_{A}^{\perp}(\theta) D(\theta)\right\}^{-1} \\
& \operatorname{CRB}(\mathbf{x})=\sigma_{e}^{2}\left\{A^{T}(\theta) P_{D}^{\perp}(\theta) A(\theta)\right\}^{-1} \\
& \operatorname{CRB}\left(\sigma_{e}^{2}\right)=\frac{2 \sigma_{e}^{4}}{m p}
\end{aligned}
$$

where $D(\theta)=\partial \mathbf{a}(\theta) / \partial \theta$ is an $(m p \times 5)$-dimensional matrix. These are derived in detail in [24].

\section{Modified FIM Motion Algorithm}

In this section we briefly introduce the Modified FIM Motion Algorithm. Please see [23] for the full details.

Suppose the sensor has taken $n$ measurements at $n$ known locations and instants. Assume the measurements were taken periodically with period $T$ so that the time to take $n$ measurements is $n T$. With these $n$ measurements, an estimate $\left[\hat{x}_{\circ n}, \hat{y}_{\circ n}\right]^{T}$ of the source coordinates can be obtained using maximum likelihood estimation. The accuracy of these estimates can be estimated from the Cramér-Rao lower bound if we assume that the algorithm is statistically efficient (or the variances of the estimates are a small multiple of the bound, if we allow for relative efficiency) [25]. While the CRB depends on the unknown parameters $\theta$ so it can not be computed, an estimate of the CRB can be obtained by substituting the current estimates for all unknown parameters. We first compute the Fisher Information Matrix (FIM) which has the following form:

$$
\operatorname{FIM}(\Psi)=\frac{1}{\sigma_{e}^{2}}\left[\begin{array}{ccc}
\mu^{2} D^{T}(\theta) D(\theta) & \mu A^{T}(\theta) A(\theta) & 0 \\
\mu A^{T}(\theta) D(\theta) & A^{T}(\theta) A(\theta) & 0 \\
0 & 0 & \frac{n}{2 \sigma_{e}^{2}}
\end{array}\right] .
$$

For a general parameter vector $\xi$, we may partition the parameter space as $\xi=\left[\xi_{1}, \xi_{2}\right]^{T}$ where $\xi_{1}$ is a vector containing the parameters of interest, and $\xi_{2}$ is a vector containing all nuisance parameters. Then, with the probability density function $p(\mathbf{y} ; \xi)$ given, the $\operatorname{FIM}(\xi)$ has the following structure:

$$
\operatorname{FIM}(\xi)=\left[\begin{array}{ll}
-E\left[\frac{\partial^{2} \ln p(\mathbf{y} ; \xi)}{\partial \xi_{1} \partial \xi_{1}^{T}}\right] & -E\left[\frac{\partial^{2} \ln p(\mathbf{y} ; \xi)}{\partial \xi_{1} \partial \xi_{2}^{T}}\right] \\
-E\left[\frac{\partial^{2} \ln p(\mathbf{y} ; \xi)}{\partial \xi_{2} \partial \xi_{1}^{T}}\right] & -E\left[\frac{\partial^{2} \ln p(\mathbf{y} ; \xi)}{\partial \xi_{2} \partial \xi_{2}^{T}}\right]
\end{array}\right] .
$$

Using the formula for inverting block matrices [25], $\mathrm{CRB}\left(\xi_{1}\right)$ is

$$
\begin{aligned}
& C R B\left(\xi_{1}\right)=\left(-E\left[\frac{\partial^{2} \ln p(\mathbf{y} ; \xi)}{\partial \xi_{1} \partial \xi_{1}^{T}}\right]+\right. \\
& \left.E\left[\frac{\partial^{2} \ln p(\mathbf{y} ; \xi)}{\partial \xi_{1} \partial \xi_{2}^{T}}\right] E\left[\frac{\partial^{2} \ln p(\mathbf{y} ; \xi)}{\partial \xi_{2} \partial \xi_{2}^{T}}\right]^{-1} E\left[\frac{\partial^{2} \ln p(\mathbf{y} ; \xi)}{\partial \xi_{2} \partial \xi_{1}^{T}}\right]\right)^{-1}
\end{aligned}
$$

As can be seen from the equation, using the true CRB for motion control adds extra complexity to the stability analysis since it involves terms unrelated to the location coordinates of the chemical source, which are the parameters of interest. For this reason, we will devise a motion algorithm based on the upper left-hand block of $\operatorname{FIM}(\Psi)$.

The upper left-hand block of FIM $(\Psi)$ pertains to the Fisher information of the parameter vector $\theta=\left[x_{\circ}, y_{\circ}, \kappa, t_{\circ}\right]^{T}$. We will partition this block even further to remove the effects of the nuisance parameters $\left[\kappa, t_{\circ}\right]^{T}$. The piece of interest has the following form:

$$
\frac{\mu^{2}}{\sigma_{e}^{2}}\left[\begin{array}{ll}
\mathbf{d}_{1}^{T} \mathbf{d}_{1} & \mathbf{d}_{1}^{T} \mathbf{d}_{2} \\
\mathbf{d}_{2}^{T} \mathbf{d}_{1} & \mathbf{d}_{2}^{T} \mathbf{d}_{2}
\end{array}\right]=D_{1}^{T}(\theta) D_{1}(\theta)
$$

where $\mathbf{d}_{1}$ and $\mathbf{d}_{2}$ are the first two columns of $D(\theta)$ and $D_{1}(\theta)$ is an $n \times 2$ matrix of the form $\left[\mathbf{d}_{1} \mathbf{d}_{2}\right]$.

If we compute the gradient of the inverse of (12) after $n+1$ measurements, and move the sensor in the opposite direction to the gradient we are guaranteed to have an equilibrium point at the source location. Going through the same process as in [23], the gradient of (12) is found to be

$$
\begin{aligned}
& \frac{\partial}{\partial \zeta} \frac{\mu^{2}}{\sigma_{e}^{2}}\left[D_{1}^{T}(\theta) D_{1}(\theta)\right]^{-1}=-\frac{\mu^{2}}{\sigma_{e}^{2}}\left[D_{1}^{T}(\theta) D_{1}(\theta)\right]^{-1} . \\
& {\left[\frac{\partial D_{1}^{T}(\theta)}{\partial \zeta} D_{1}(\theta)+D_{1}^{T}(\theta) \frac{\partial D_{1}(\theta)}{\partial \zeta}\right] \cdot\left[D_{1}^{T}(\theta) D_{1}(\theta)\right]^{-1} .}
\end{aligned}
$$

To determine the direction of motion of the sensor, we must devise a scalar criterion. It was shown in [23] that the gradient will be zero at the source location no matter which elements we choose, and thus we have chosen the trace of (13). The motion algorithm is thus:

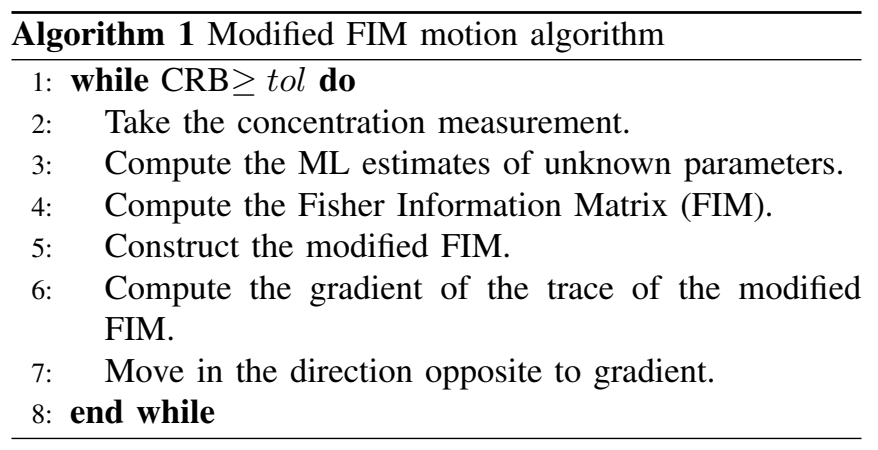

Unfortunately, since the matrix $D_{1}(\theta)$ depends on the history of the sensor's motion, it is not possible to determine analytically through linearization the stability properties of the equilibrium point at the center of the vapor source. However we can show through the properties of ML estimates that the center of the vapor source is the only equilibrium point.

\section{Stability Analysis Through Consistency}

In this section we prove that the center of the circular source is the only equilibrium point of the system. Previous attempts at proving this point were difficult since the gradient of the motion algorithm employed, namely the direction of gradient algorithm [15], contained elements pertaining to parameters that were not of interest to us. As was shown 
in [23], removal of these elements allowed us to show that the location of the source was an equilibrium point of the system. We will now show formally through a combination of control theory and statistical analysis that this is the only equilibrium point of the system.

An estimator is said to be consistent if it approaches its true value in probability [26]. In other words,

$$
\lim _{n \rightarrow \infty} P[|\hat{\theta}-\theta|>\epsilon]=0 \quad \forall \epsilon>0 \text {. }
$$

We will show here that this is the case for our estimate $\hat{\theta}$. Recall that our estimate of $\sigma_{e}^{2}$ was

$$
\hat{\sigma}_{e}^{2}=\frac{1}{n} \mathbf{y}^{T} P_{A}^{\perp}(\hat{\theta}) \mathbf{y} .
$$

Through direct comparison it can be seen that (15) is equivalent to

$$
\operatorname{tr}\left\{P_{A}^{\perp}(\hat{\theta}) \hat{R}\right\}
$$

where $\hat{R}=\frac{1}{n} \mathbf{y} \mathbf{y}^{T}$. Inserting (15) and (7) into the loglikelihood function

$\ln L(\mathbf{y} \mid \Psi)=\mathrm{const}-n \ln \sigma_{e}^{2}-\frac{1}{\sigma_{e}^{2}}(\mathbf{y}-A(\theta) \mathbf{x})^{T}(\mathbf{y}-A(\theta) \mathbf{x})$

we obtain

$$
\ln L(\mathbf{y} \mid \Psi)=\mathrm{const}-n \ln \operatorname{tr}\left\{P_{A}^{\perp}(\hat{\theta}) \hat{R}\right\} .
$$

Therefore the ML estimate of $\theta$ is the minimizer of

$$
\operatorname{tr}\left\{P_{A}^{\perp}(\hat{\theta}) \hat{R}\right\} \text {. }
$$

Letting $n \rightarrow \infty$, we arrive to the following inequality

$$
\begin{aligned}
& \operatorname{tr}\left\{P_{A}^{\perp}(\hat{\theta}) \hat{R}\right\} \\
& =\operatorname{tr}\left\{P_{A}^{\perp}(\hat{\theta}) R\right\} \quad \text { by Law of Large Numbers } \\
& =\operatorname{tr}\left\{P_{A}^{\perp}(\hat{\theta})\left[A(\theta) \mathbf{x} \mathbf{x}^{T} A^{T}(\theta)+\sigma_{e}^{2} I\right]\right\} \\
& =\operatorname{tr}\left\{P_{A}^{\perp}(\hat{\theta}) A(\theta) \mathbf{x} \mathbf{x}^{T} A^{T}(\theta)+\sigma_{e}^{2} I-\sigma_{e}^{2} P_{A}(\theta)\right\} \\
& =\operatorname{tr}\left\{P_{A}^{\perp}(\hat{\theta}) A(\theta) \mathbf{x} \mathbf{x}^{T} A^{T}(\theta)\right\}+n \sigma_{e}^{2}-\operatorname{tr}\left\{\sigma_{e}^{2} I_{2 \times 2}\right\} \\
& =\operatorname{tr}\left\{P_{A}^{\perp}(\hat{\theta}) A(\theta) \mathbf{x} \mathbf{x}^{T} A^{T}(\theta)\right\}+\sigma_{e}^{2}(n-2) \\
& \quad \geq \sigma_{e}^{2}(n-2) .
\end{aligned}
$$

By expanding out

$$
\begin{aligned}
\operatorname{tr}\left\{P_{A}^{\perp}(\hat{\theta}) A(\theta) \mathbf{x} \mathbf{x}^{T} A^{T}(\theta)\right\}= \\
\operatorname{tr}\left\{\left[I-\hat{A}\left(\hat{A}^{T} \hat{A}\right)^{-1} \hat{A}^{T}\right] A \mathbf{x} \mathbf{x}^{T} A^{T}\right\}
\end{aligned}
$$

and using the shorthand notation $\hat{A}=A(\hat{\theta})$ it is clear that (19) is minimized by $A(\hat{\theta})=A(\theta)$, which shows that $\hat{\theta}$ is consistent when $n \rightarrow \infty$.

The trace of (13) can be written as

$$
\begin{aligned}
& \left(\frac{\partial \mathbf{d}_{1}^{T}}{\partial \zeta} \mathbf{d}_{1}+\frac{\partial \mathbf{d}_{2}^{T}}{\partial \zeta} \mathbf{d}_{2}\right)\left(\left(\mathbf{d}_{1}^{T} \mathbf{d}_{1}\right)^{2}+\left(\mathbf{d}_{2}^{T} \mathbf{d}_{2}\right)^{2}\right) \\
& -\left(\frac{\partial \mathbf{d}_{2}^{T}}{\partial \zeta} \mathbf{d}_{1}+\frac{\partial \mathbf{d}_{1}^{T}}{\partial \zeta} \mathbf{d}_{2}\right)\left(\mathbf{d}_{2}^{T} \mathbf{d}_{2} \mathbf{d}_{1}^{T} \mathbf{d}_{2}+\mathbf{d}_{1}^{T} \mathbf{d}_{1} \mathbf{d}_{1}^{T} \mathbf{d}_{2}\right)
\end{aligned}
$$

For a sensor location $\vec{r}$ to be an equilibrium point, the gradient at that location must be zero for all times. Due to the consistency of $\hat{\theta}$, it is enough to show that at any time $t$, and $\hat{\theta}=\theta$, the gradient is zero only when $\vec{r}=\vec{r}_{\mathrm{o}}$. This

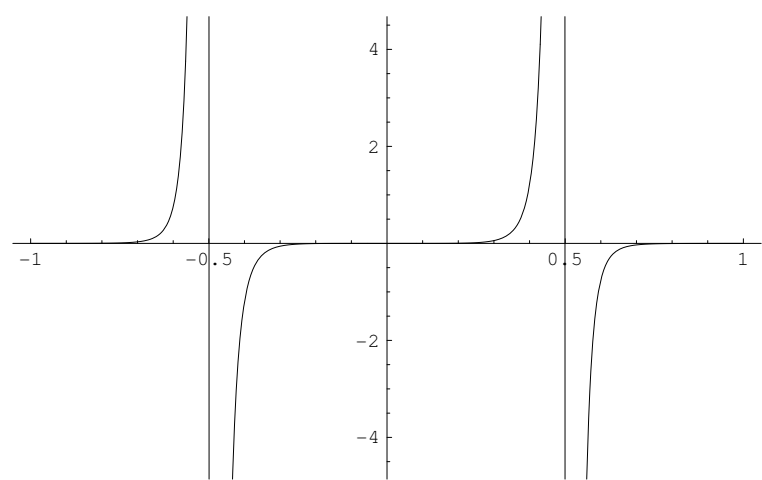

Fig. 2. An illustration of the motion gradient, with $\hat{\theta}=\theta$. The center of the vapor source is assumed to be at the origin, and the vapor source has a radius of 0.5 .

is shown in Figure 2. Because of the circular symmetry of the concentration function, a one dimensional illustration is all that is necessary to illustrate the point. As can be seen in the figure, the only point at which the gradient is zero is the center of the vapor source. Thus, in the limit, as $n \rightarrow \infty$, only the point $\vec{r}=\vec{r}_{\circ}$ is an equilibrium point. Furthermore, it is an unstable equilibrium point. Also notice that at every point outside of the source, this motion algorithm drives the vehicle towards the source, but not to the center of the source. From Figure 2 it should be expected that as $\hat{\theta}$ converges, the vehicle should be driven to the edge of the source.

This algorithm can be extended to the multiple moving sensor case very easily. Using a distributed network, all sensors transmit to their neighbors their current concentration measurement. A neighbor can be considered to be any sensor within a given communication radius for example. Each sensor then computes their own ML estimates and employs the modified FIM motion algorithm to determine its next location. Once that location is reached, each sensor determines who its new neighbors are, and then repeats the process. This process is continued until one of the sensors estimates the source location with some predetermined level of accuracy. An example of such an approach is given in [22].

\section{RESUlTS}

In this section we employ the modified FIM gradient algorithm. A vapor emitting source is placed at the coordinates $(-50,-50) \mathrm{m}$ and has a radius of $0.5 \mathrm{~m}$. The diffusion rate $\mu$ and diffusivity $\kappa$ are set to $10 \mathrm{Kg} / \mathrm{s}$ and $25 \mathrm{~m}^{2} / \mathrm{s}$ respectively. A bias $b$ of $10^{-4} \mathrm{Kg} / \mathrm{m}^{3}$ and zero-mean Gaussian noise with standard deviation, $\sigma=10^{-8} \mathrm{Kg} / \mathrm{m}^{3}$ are considered to be present in the measurements. The emission of vapor begins $100 \mathrm{~s}$ before any measurements are taken. Beginning at $t=100 \mathrm{~s}$ the vehicle starts moving in a random path starting at a distance of $200 \mathrm{~m}$ from the origin at a speed of $2.5 \mathrm{~m} / \mathrm{s}$ and collects measurements every $10 \mathrm{~s}$.

The detection of the vapor is assumed to have occurred at $t=390 \mathrm{~s}$ and the modified FIM gradient algorithm begins, using the measurements obtained from a random walk. In this phase, the vehicle moves at a maximum speed of $2.5 \mathrm{~m} / \mathrm{s}$, and a line search algorithm is employed to find the minimum along the direction of the gradient up to the maximum 


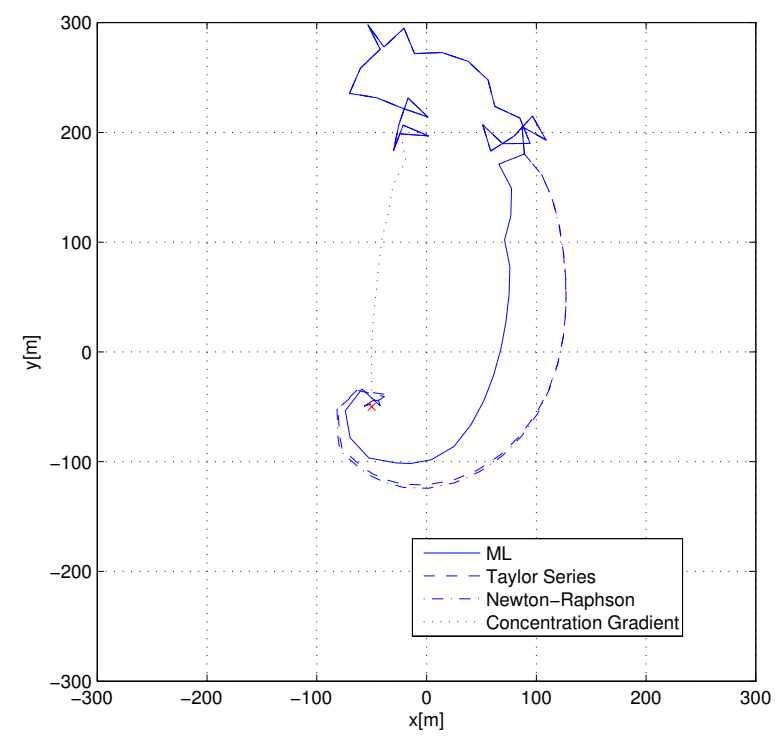

Fig. 3. The sensor's motion using maximum likelihood, Taylor series, and Newton-Raphson methods for parameter estimation. The sensor's motion is also illustrated for concentration gradient driven motion.

distance the vehicle can travel, which is $25 \mathrm{~m}$. The motion algorithm stops once the CRB for both position estimates goes below $10^{-6} \mathrm{~m}^{2}$.

Figure 3 illustrates the movement of the sensor using the modified FIM gradient algorithm, using the different estimation methods presented in previous work [22]. In each case the sensor takes a circuitous path towards the source, obtaining spatially diverse measurements that help in pinpointing the exact location of the source. These results are quite similar to the ones in [15] where the Direction of Gradient (DOG) algorithm was employed. When maximum likelihood estimation was used, a total of $290 \mathrm{~s}$ were needed for the vehicle to complete the task. In the cases where Taylor Series and Newton-Raphson estimation were used, a total of $400 \mathrm{~s}$ and $280 \mathrm{~s}$ were needed respectively to complete the task. It should be noted that in terms of real time needed for the simulations to finish, both the Taylor Series and Newton-Raphson simulations finished in under $5 \mathrm{~min}$, while the ML approach took over $20 \mathrm{~min}$. This is due to the fact that to compute the maximum likelihood estimates, one needs to perform a nonlinear search which is computationally expensive.

The first inset in Figure 4 illustrates the estimate of the CRB of the position estimates for each of the estimation methods. We have plotted the sum of the square roots of the CRB's of the position estimates on a log scale. The CRB's in all cases decrease monotonically in general, as in [15] where the DOG motion algorithm was used with the tactic of minimizing the CRB at every step.

The second and third insets in Figure 4 shows the distance between the current estimate of the source position and the source. In each case the algorithm converges to the source location. In the case of maximum likelihood estimation, the estimate is poor at first and hence a very high CRB estimate. It may seem that this is a negative aspect of this approach, however it is our experience that when the estimates become poor for the other two estimation approaches, they almost never converge to the correct value. It is very well known that when someone uses the Newton-Raphson method to find minima or zero crossings of a function, a good initial guess is necessary so that one does not fall into local minima or move towards another zero crossing of the function. Likewise for the Taylor series method, a poor estimate of $\theta$ will give a very poor fit between the model matrix $A(\theta)$ and the data vector $\mathbf{y}$.

\section{A. Gradient driven robot motion}

A number of previous approaches to locating a vapor source rely on estimating the local concentration gradient and moving the robot in the direction of the gradient's increase [10]:

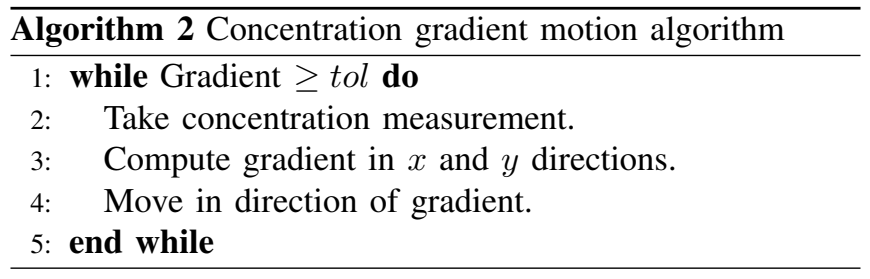

In practice the robot is equipped with two or more physically separated sensing units. In most cases it is assumed that there exists wind, thereby making the concentration gradient more pronounced. We performed a simulation of a robot locating a vapor source with this technique. We assume our robot is equipped with four chemical sensors, each $90^{\circ}$ from its nearest neighbor, and $38 \mathrm{~cm}$ away from the sensor that is its diametric opposite. This distance is the diameter of the Super Scout II robot which is commonly used in sensing task experiments.

To account for the presence of wind, the diffusion equation (1) takes the form [4]

$$
\frac{\partial c}{\partial t}=\kappa \nabla^{2} c-\nabla \cdot(c \vec{v})
$$

where $\vec{v}$ is the wind speed vector. The solution to (21) is given by

$$
\begin{aligned}
& c(\vec{r}, t)=\frac{\mu}{8 \pi \kappa\left|\vec{r}-\vec{r}_{\circ}\right|} e^{\frac{\left(\vec{r}-\vec{r}_{\circ}\right) \cdot \vec{v}}{2 \kappa} .} \\
& {\left[e^{\frac{\left|\vec{r}-\vec{r}_{\circ}\right||\vec{v}|}{2 \kappa}} \times \operatorname{erfc}\left(\frac{\left|\vec{r}-\vec{r}_{\circ}\right|}{2 \sqrt{\kappa\left(t-t_{\circ}\right)}}+|\vec{v}| \sqrt{\frac{t-t_{\circ}}{4 \kappa}}\right)+\right.} \\
& \left.e^{-\frac{\left|\vec{r}-\vec{r}_{\circ}\right||\vec{v}|}{2 \kappa}} \times \operatorname{erfc}\left(\frac{\left|\vec{r}-\vec{r}_{\circ}\right|}{2 \sqrt{\kappa\left(t-t_{\circ}\right)}}-|\vec{v}| \sqrt{\frac{t-t_{\circ}}{4 \kappa}}\right)\right] .
\end{aligned}
$$

A wind speed of $1 \mathrm{~m} / \mathrm{s}$ in the $+y$ direction is used in the simulation. All other parameters are as stated at the beginning of this section.

A plot of the trajectory taken by the robot is given in Figure 3 to compare to the trajectories using the modified FIM algorithm. Using the concentration gradient technique, it takes the robot $100 \mathrm{~s}$ to reach the vapor source, and its path to the source is much more straightforward. The speed at which it finishes the task can be attributed to its simplicity. The 

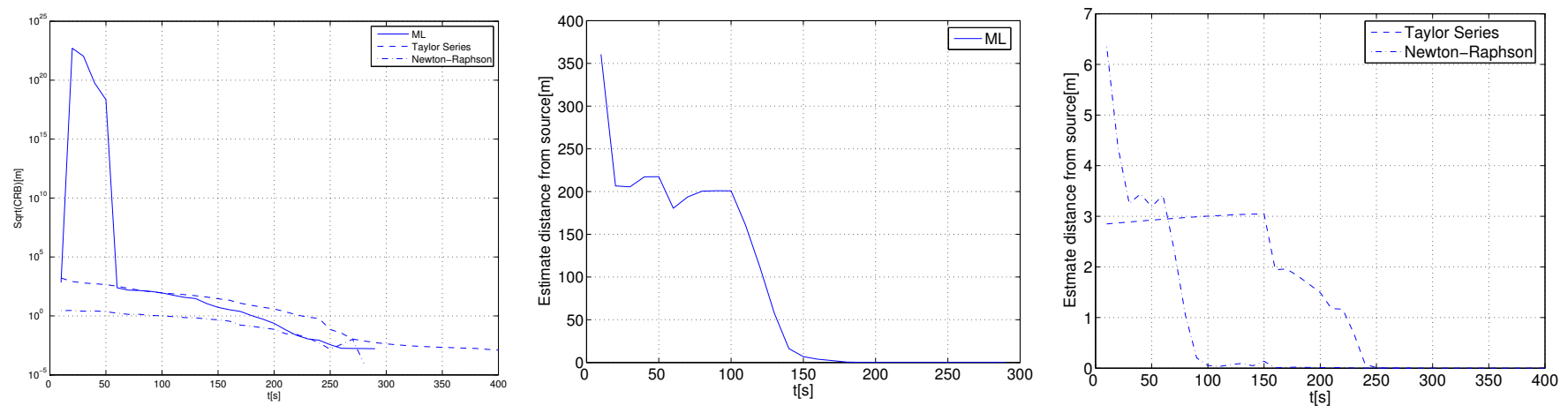

Fig. 4. Cramér-Rao bounds on the source location coordinates, and source location estimates as a function of time.

only calculations that it makes is computing the difference in the concentration levels between the diametrically opposed sensors. On the other hand, the modified FIM motion algorithm is much more complicated. At each step, it must perform numerical integration, multiple matrix inversions, and possibly a nonlinear search for the unknown parameters. The concentration gradient technique may be fast, however it does not perform any estimation of the source location, and therefore cannot be used in situations in which the source must be located remotely.

\section{CONCLUSION}

In this paper we have applied our modified FIM motion algorithm to the task of locating a circular chemical source. We provide the diffusion model for a circular source and prove that due to consistency of the maximum likelihood (ML) estimator, the only equilibrium point of the system is the center of the circular source. Simulations demonstrate that the robot is successful in locating the source using this motion algorithm. While modeling a chemical source as a circular source rather than a point source is certainly more realistic, the model is not applicable to realistic settings such as urban, or indoor environments. Future work entails combining our motion algorithm with numerical modeling of realistic environments to obtain methods that are both robust and practical.

\section{REFERENCES}

[1] A. Jeremić and A. Nehorai, "Landmine detection and localization using chemical sensor array processing," IEEE Trans. Signal Processing, vol. 48, no. 5, pp. 1295-1305, 2000.

[2] E.A. Kaltenbacher, R.H. Byrne, and E.T. Steimle, "Design and applications of a chemical sensor compatible with autonomous oceansampling networks," IEEE J. Oceanic Eng., vol. 26, no. 4, pp. 667670, 2001

[3] J.P. Fitch, E. Raber and D.R. Imbro, "Technology challenges in responding to biological or chemical attacks in the civilian sector," Science, vol. 32, pp. 1350-1354, 2003.

[4] A. Nehorai, B. Porat, and E. Paldi, "Detection and localization of vapor-emitting sources," IEEE Trans. Signal Processing, vol. 43, no. 1, pp. 243-253, 1995.

[5] J. Cortés, S. Martínez, T. Karatas, and F. Bullo, "Coverage control for mobile sensing networks," IEEE Trans. Robotics and Automation, vol. 20, no. 2, pp. 243-255, 2004.

[6] D. Estrin, R. Govindan, J. Heidemann, and S. Kumar, "Next century challenges: scalable coordination in sensor networks," in Fifth Annual International Conference on Mobile Computing and Networks, 1999.

[7] H. Ishida, T. Nakamoto, and T. Morizumi, "Remote sensing of gas/odor source location and concentration distribution using mobile system," Sensors and actuators B, vol. 49, no. 1-2, pp. 52-57, 1998.
[8] F. W. Grasso, "Invertebrate-inspired sensory-motor systems and autonomous, olfactory-guided exploration," Biological bulletin, vol. 200, no. 2, pp. 160-168, 2001.

[9] R. A. Russell, "Survey of robotic applications for odor-sensing technology," International Journal of Robotics Research, vol. 20, no. 2, pp. 144-162, 2001.

[10] L. Marques, U. Nunes, and A. de Almeida, "Olfaction-based mobile robot navigation," Thin solid films, vol. 418, no. 1, pp. 51-58, 2002.

[11] S. Kazadi, R. Goodman, D. Tsikata, D. Green, and H. Lin, "An autonomous water vapor plume tracking robot using passive resistive polymer sensors," Autonomous robots, vol. 9, no. 2, pp. 175-188, 2000.

[12] A.T. Hayes, A. Martinoli, and R.M. Goodman, "Swarm robotic odor localization," in IEEE?RSJ Int. Conf. on Intelligent Robots and Systems, 2001, pp. 1073-1078.

[13] T. M. Morse, T. C. Ferrée, and S. R. Lockery, "Robust spatial navigation in a robot inspired by chemotaxis in caenorhabditis elegans," Adapt. Behav., vol. 6, no. 3-4, pp. 393-410, 1998.

[14] G. Sandini, G. Lucarini, and M. Varoli, "Gradient driven selforganizing systems," in IEEE?RSJ Int. Conf. on Intelligent Robots and Systems, 1993, pp. 429-432.

[15] B. Porat and A. Nehorai, "Localizing vapor-emitting sources by moving sensors," IEEE Trans. Signal Processing, vol. 44, no. 4, pp. 1018-1021, 1996.

[16] J.P. Helferty and D.R. Mudgett, "Optimal observer trajectories for bearings-only tracking by minimizing the trace of the Crámer-Rao lower bound," in IEEE Conf. on Decision and Control, 1993, pp. 936939.

[17] D. Uciński, "Optimal sensor location for parameter estimation of distributed processes," Int. J. of Control, vol. 73, no. 13, pp. 1235$1248,2000$.

[18] T.H. Chung, V. Gupta, J.W. Burdick, and R.M. Murray, "On a decentralized active sensing strategy using mobile sensor platforms in a network," in IEEE Conf. on Decision and Control, 2004, pp. 1914-1919.

[19] V.N. Christopoulos and S. Roumeliotis, "Adaptive sensing for instantaneous gas release parameter estimation," in IEEE Int. Conf. on Robotics and Automation, 2005, pp. 4461-4467.

[20] J. Crank, The Mathematics of Diffusion. Oxford, UK: Oxford University Press, 1956.

[21] H.S. Carslaw and J.C. Jaeger, Conduction of Heat in Solids. New York: Oxford University Press, 1959.

[22] P. Tzanos, M. Žefran, and A. Nehorai, "Information based distributed control for biochemical source detection and localization," in IEEE Int. Conf. on Robotics and Automation, 2005, pp. 4468-4473.

[23] P. Tzanos and M. Žefran, "Stability analysis of information based control for biochemical source localization," in IEEE Int. Conf. on Robotics and Automation, 2006.

[24] P. Stoica and A. Nehorai, "MUSIC, maximum likelihood, and CramérRao bound," IEEE Trans. Acoust., Speech, Signal Processing, vol. 37, no. 5, pp. 720-741, 1989.

[25] S. M. Kay, Fundamentals of Statistical Signal Processing: Estimation Theory. Upper Saddle River, NJ: Prentice Hall, 1993.

[26] H. Stark and J. W. Woods, Probability, Random Processes, and Estimation Theory for Engineers. Upper Saddle River, NJ: Prentice Hall, 1994. 